\title{
Human metapneumovirus prevalence and patterns of subgroup persistence identified through surveillance of pediatric pneumonia hospital admissions in coastal Kenya, 2007-2016
}

John W. Oketch ${ }^{1 *}$ (D), Everlyn Kamau', Grieven P. Otieno ${ }^{1}$, James R. Otieno ${ }^{1}$, Charles N. Agoti ${ }^{1,2}$ and D. James Nokes ${ }^{1,2,3}$

\begin{abstract}
Background: Human metapneumovirus (HMPV) is an important respiratory pathogen that causes seasonal epidemics of acute respiratory illness and contributes significantly to childhood pneumonia. Current knowledge and understanding on its patterns of spread, prevalence and persistence in communities in low resource settings is limited.

Methods: We present findings of a molecular-epidemiological analysis of nasal samples from children $<5$ years of age admitted with syndromic pneumonia between 2007 and 2016 to Kilifi County Hospital, coastal Kenya. HMPV infection was detected using real-time RT-PCR and positives sequenced in the fusion (F) and attachment (G) genes followed by phylogenetic analysis. The association between disease severity and HMPV subgroup was assessed using Fisher's exact test.

Results: Over 10 years, 274/6756 (4.1\%) samples screened were HMPV positive. Annual prevalence fluctuated between years ranging 1.2 to $8.7 \%$ and lowest in the recent years (2014-2016). HMPV detections were most frequent between October of one year to April of the following year. Genotyping was successful for 205/274 (74.8\%) positives revealing clades A2b (41.0\%) and A2c (10.7\%), and subgroups B1 (23.4\%) and B2 (24.9\%). The dominance patterns were: clade A2b between 2007 and 11, subgroup B1 between 2012 and 14, and clade A2c in more recent epidemics. Subgroup B2 viruses were present in all the years. Temporal phylogenetic clustering within the subgroups for both local and global sequence data was seen. Subgroups occurring in each epidemic season were comprised of multiple variants. Pneumonia severity did not vary by subgroup $(p=0.264)$. In both the $F$ and $G$ gene, the sequenced regions were found to be predominantly under purifying selection.

Conclusion: Subgroup patterns from this rural African setting temporally map with global strain distribution, suggesting a well-mixed global virus transmission pool of HMPV. Persistence in the local community is characterized by repeated introductions of HMPV variants from the global pool. The factors underlying the declining prevalence of HMPV in this population should be investigated.
\end{abstract}

Keywords: Human metapneumovirus, Prevalence, Subgroup, Epidemic, Temporal

\footnotetext{
* Correspondence: joketch@kemri-wellcome.org; oketchjohn9@gmail.com

${ }^{1}$ Kenya Medical Research Institute (KEMRI) -Wellcome Trust Research

Programme, Kilifi, KEMRI Centre for Geographic Medicine Research - Coast,

Kilifi, Kenya

Full list of author information is available at the end of the article
}

(c) The Author(s). 2019 Open Access This article is distributed under the terms of the Creative Commons Attribution 4.0 International License (http://creativecommons.org/licenses/by/4.0/), which permits unrestricted use, distribution, and reproduction in any medium, provided you give appropriate credit to the original author(s) and the source, provide a link to the Creative Commons license, and indicate if changes were made. The Creative Commons Public Domain Dedication waiver (http://creativecommons.org/publicdomain/zero/1.0/) applies to the data made available in this article, unless otherwise stated. 


\section{Background}

Human metapneumovirus (HMPV) is single-stranded, negative-sense RNA virus with a genome of about $13 \mathrm{~kb}$ $[1,2]$. The virus belongs to the Pneumoviridae family and genus Metapneumovirus. HMPV infections occur across all ages with severe disease predominantly occurring in children below 2 years of age and the elderly [35]. Since first description in 2001 [5], HMPV has been detected in all continents and its disease prevalence varies widely [6]. Nearly every child by the age of 5 years has been infected by HMPV [5, 7]. Clinical presentation of HMPV infection ranges from mild upper respiratory tract illness to severe lower respiratory tract disease [8] and overlaps with that of other common respiratory viruses especially respiratory syncytial virus (RSV) $[9,10]$.

HMPV is classified into two major groups, A and B, based on antigenic variation and nucleotide differences in the fusion (F), nucleoprotein (N) and attachment (G) glycoprotein genes $[2,11,12]$. Phylogenetic analyses of $\mathrm{F}$ (open reading frame, i.e. ORF) and $G$ (ORF) sequences further divides the two groups into subgroups i.e. A1 and A2 (group A), and B1 and B2 (group B) subgroups $[11,13]$. In addition, there has been report of within subgroup A2 division, i.e. presence of two phylogenetically distinct clades A2a and A2b [14]. A few studies have revealed increased heterogeneity of $\mathrm{A} 2$, including identification of another distinct clade, provisionally assigned as A2c $[15,16]$. Furthermore, two novel clades within subgroup A2 with 180 or 111 nucleotide duplications in the $\mathrm{G}$ gene have been detected [17-20]. Geographically, HMPV subgroups have been reported to circulate widely and cluster temporally [21], with the exception of subgroup A1 that has been identified in a few countries [21-23]. There is frequent co-circulation of subgroups with replacement of the predominant subgroup after a period of one or two seasons, although the drivers of this phenomena are unclear [21]. Virus prevalence also varies from year-to-year within the same location [24, 25].

A few studies have reported long-term subgroup circulation patterns of HMPV, necessary for improved epidemiological understanding and, in due course, of potential value to the design and implementation of control measures. In addition, there are a few studies on HMPV in Africa, which bears a high burden of pneumonia morbidity and mortality [26, 27]. In this study, we analysed the surface $F$ and $G$ gene nucleotide sequences collected through paediatric pneumonia surveillance at the Kilifi County Hospital in rural coastal Kenya, from 2007 to 2016, to describe the molecular epidemiology and gain insights into the spread and the evolutionary dynamics of HMPV. The two genes ( $F$ and $G$ ) code for immunogenic surface proteins that are the targets for vaccine development. $G$ gene is highly variable and is used to assess the diversity of HMPV. Similarly, F gene which is quite conserved, has been used to characterise HMPV and is the gene of target in many molecular diagnostic assays. This report extends our previously published work from the same location for the period 2007-11 [28] and the analysis includes sequences from this previous work.

\section{Methods \\ Study population}

The study was conducted at the Kilifi County Hospital $(\mathrm{KCH})$ as part of long-term surveillance aimed at understanding the epidemiology and disease burden of RSV-associated pneumonia cases [29]. $\mathrm{KCH}$, located in coastal Kenya, is a referral hospital serving the population of around 260,000 (circa 2012) within the Kilifi Health and Demographic Surveillance System [30], and beyond in the wider Kilifi County. The population is mainly rural-agrarian. Upon presentation of a child to the paediatric ward at $\mathrm{KCH}$, a detailed medical review is undertaken by the clinician upon which the decision to admit is made. For this study, children ( $\leq 59$ months of age) admitted to the paediatric ward between January 1st 2007 and December 31st 2016 were eligible if they presented with modified WHO defined syndromic severe or very severe pneumonia as previously described [29]. During the period between 2007 and 2009, only admissions arising from residents of Kilifi Health and Demographic Surveillance System (KHDSS) were eligible, whereas in later years, non-KHDSS residents were included. Following written informed consent from the parent or guardian, a nasopharyngeal flocked swab, nasal wash or combination of nasopharyngeal swab and oropharyngeal swab was collected from each child and transferred into viral transport medium for laboratory screening. Ethical approval for the study was obtained from the Kenya Medical Research Institute Scientific and Ethics Review Unit.

\section{RNA extraction and rRT-PCR}

For samples collected from 2007 to 2011 the methods for extraction and detection were as previously described [31, 32]. In brief, for samples collected in 2007 RNA was extracted using Magnapure LC32 total nucleic acid extractor (Roche, Manheim, Germany) and virus screening conducted using the LightCycler Fast Start DNA MasterPLUS Hyb-Probe kit (Roche, Mannheim, Germany) following manufacturer's instructions. From 2008 to 2011, sample extraction was by MagNaPure LC32 Kit (Roche, Manheim, Germany) or QIAmp Viral RNA Minikit (Qiagen, Germany) and virus detection carried out using a one-step in-house multiplex real time reverse transcription-PCR (rRT-PCR) using a TaqMan probe-based system targeting the fusion (F) gene [28, 32]. Samples with a rRT-PCR cycle threshold $(\mathrm{Ct})$ value 
$<35$ were considered HMPV positive. From 2012 to 2016 total RNA extraction was performed using Qiacube HT automated total nucleic acid extractor (Qiagen, Germany) and virus detection carried out using the same one-step in-house multiplex real time reverse transcription-PCR (rRT-PCR) used for samples from 2008 to 11 [28].

\section{$F$ and $G$ gene sequencing}

HMPV positive samples identified between 2007 and $2011(n=160)$ were partially sequenced in the F and G genes, 345 and $606 \mathrm{bp}$, respectively [28]. For HMPV positive samples identified between 2012 and $2016(n=$ 114 ), we amplified full $\mathrm{F}$ and $\mathrm{G}$ encoding genes, approximately $1620 \mathrm{bp}$ and $700 \mathrm{bp}$, respectively, using one-step RT-PCR kit (Qiagen, Germany) according to manufacturer's instructions. RT-PCR and sequencing primers are shown in Additional file 1. Thermocycling conditions for both genes were set at: $50^{\circ} \mathrm{C}$ for $30 \mathrm{~min}, 95^{\circ} \mathrm{C}$ for 15 min, 38 cycles of $94{ }^{\circ} \mathrm{C}$ for $1 \mathrm{~min}, 53^{\circ} \mathrm{C}$ for $1 \mathrm{~min}$, $72^{\circ} \mathrm{C}$ for $1 \mathrm{~min}$, and a final extension of $10 \mathrm{~min}$ at $72^{\circ} \mathrm{C}$. HMPV A and B subtype specific primers were used to increase the sensitivity and the performance of $\mathrm{G}$ gene amplification. HMPV positive samples were sequenced in both forward and reverse directions using Big dye terminator v1.3 chemistry run on $\mathrm{ABI}$ 3130xl instrument [33].

\section{Sequence analysis and diversity}

Sequence reads for the successfully amplified HMPV positives identified between 2012 to 2016 were edited and assembled using Sequencher v5.0.1 (Gene Codes Corporation). The newly assembled $\mathrm{F}$ and $\mathrm{G}$ genes sequences were deposited in GenBank under accession numbers MH482553 to MH482743. The accession numbers (MH482553 to MH482743) include additional 31 sequences that were detected by different rRT-PCR assay and therefore they were excluded in this analysis. The accession numbers for the excluded sequences (15 for $\mathrm{F}$ gene and 16 for G gene) are given in Additional file 2 . For subsequent analyses, previously sequenced data for HMPV positives identified between 2007 and 2011 i.e. F $(n=123)$ and $\mathrm{G}(n=56)$ genes [28] (accession numbers KT191299 to KT191484) was combined with the newly sequenced data, making a total of 210 sequences for $F$ gene and 118 sequences for $\mathrm{G}$ gene used in subsequent analyses. Sequences were aligned with MAFFT v7.220 [34]. Nucleotide and amino acid mean genetic distances within and between subgroups were determined using Kimura-2 parameter model in MEGA v7. 0.2.6 [35]. Further, to assess sequence diversity within epidemics, collated $\mathrm{G}$ gene sequences were aligned by epidemic sampling to show changes in the $G$ gene within epidemics.

\section{Phylogenetic analysis}

Contemporaneous sequences (2007 to 2016) were obtained from GenBank for both F and G genes. Sequences had to have a complete overlap in their sequenced portion with the Kilifi sequence data. Duplicate sequences were dropped. A total of $714 \mathrm{~F}$ gene sequences and 500 $\mathrm{G}$ sequences were retrieved. For phylogenetic construction, sequences were filtered per country using an inhouse ruby script to obtain only unique sequences. Unique sequences were identified as sequences that differ by at least one nucleotide from any other sequence over the sequenced region. Combined global and Kilifi sequences were aligned using MAFFT v7.220. Best fitting nucleotide substitution and site heterogeneity models were estimated in MEGA v7. 0.2.6 and phylogenetic trees inferred using Maximum Likelihood (ML) with 1000 bootstrap replicates. Reference sequences from GenBank (FJ168778 NL/94/01/B2, AY525843 HMPV NL/1/99/B1, AF371337 HMPV NL/1/00/A1, AB503857, AY530095 HMPV JP/240/03/A2b and GQ153651 HMPV CN/gz01/08/A2) were included [36]. A fragment length of $345 \mathrm{bp}$ was analysed for $\mathrm{F}$ gene and at least $640 \mathrm{bp}$ for G gene. Subgroups were confirmed if sequences clustered with the reference sequences within a major branch with $>70 \%$ bootstrap support on the ML tree. Mean nucleotide genetic distances were also determined to assess sequence similarity between Kilifi and the global data set. To further assess the clustering of HMPV subgroups, ML tree was reconstructed using only full $\mathrm{F}(1593 \mathrm{bp})$ gene sequences.

\section{Evolutionary and selection pressure analysis of Kilfi HMPV viruses}

Substitution rates and time to the most recent common ancestor (tMRCA) were determined under uncorrelated lognormal relaxed molecular clock using Bayesian Markov Chain Monte Carlo-based approach implemented in BEAST v1.8.4. Demographic histories of HMPV subgroups were inferred using GMRF Bayesian Skyride model. BEAST analysis was set at 50 million states with sampling every 2500 steps. Selection pressure within F and $\mathrm{G}$ genes was analysed by estimating the ratios of non-synonymous to synonymous substitutions $(\mathrm{dN} / \mathrm{dS}=$ $\omega)$, using the codon-based phylogenetic method (CODEML) in PAML v 4.8a. Different site models were employed i.e. M0 (one ratio), M1a (Nearly Neutral), M2a (Positive Selection) and M3 (discrete) model. M0 model calculates a rough average value $(\omega)$ distributed across all sites (homogeneous ratios). M1a assumes two categories of sites: conserved sites with $\omega=0$ and neutral sites with $\omega=1$. M2a allows for three classes i.e. neutral $\omega=1$, purifying $(0 \leq, \omega<1)$, and positive selection $\omega>1$. M3 estimates $\omega$ ratios as M2a but using an unconstrained discrete distribution $[37,38]$. The models M0 
and M1a were used to test for selection over the entire sequenced regions while M2a and M3 tested for positively selected sites. Only sites with posterior probabilities $>95 \%$ were considered as positively selected based on Bayes Empirical Bayes method.

\section{HMPV subgroup and disease severity}

Different Kilifi HMPV subgroups identified between 2007 and 2016 were determined. To assess the association between HMPV subgroup and disease severity, we performed Fisher's exact test. Individuals were defined syndromically as either severe or very severe based on WHO definitions [39]. The clinical definitions have been described previously $[28,29,31]$. A $p$-value of $\leq 0.05$ was considered to be significant. Statistical analysis was conducted using STATA v13.1 (College Station Texas, USA).

\section{Results}

\section{HMPV prevalence in paediatric hospital admissions}

Between January 2007 and December 2016, 9079 individuals below 60 months of age were admitted to the paediatric wards at $\mathrm{KCH}$ with severe or very severe pneumonia. Samples were collected from 6756 (74\%) individuals and 274 (4.1\%) were determined HMPV positive (Table 1). Decreased HMPV prevalence was recorded in more recent years i.e. from 2014 to 2016 (Table 1). Among the 274 HMPV positive cases, $43.1 \%$ $(n=118)$ were in children $<6$ months of age and $71.5 \%$ $(n=196)$ in children $<2$ year of age. Children $\geq 3$ years accounted for only $3.6 \%$ of the cases $(n=11)$ (Table 2).

\section{HMPV subgroup assignment}

Phylogenetic analysis (F and G gene) of Kilifi, global and the reference sequences showed clustering of HMPV viruses into subgroups A2, B1 and B2. Subgroup A1 was not observed. Subgroup A2 sequences further clustered into 3 clades, the two known clades A2a and A2b, and into the unique clade A2c supported by strong bootstrap values (Figs. 1 and 2). The recently identified unique HMPV A2 strain, which possess 180 duplication in G gene clustered within A2c clade (Fig. 1) and was not observed in the Kilifi sequences. Based on $\mathrm{F}$ gene phylogeny constructed using full length coding regions, similar clades and sub-groups were also observed i.e. A2a, A2b, A2c, B1 and B2 (Additional file 3).

The prevalence of group A was 51.7\% (106/205) and group B 48.3\% (99/205). All the group A Kilifi sequences clustered within clade A2b $(84 / 106,79.2 \%)$ and within the unique clade A2c (22/106, 20.8\%). No A1 or A2a sequences were observed. Within group B, 48.5\% (48/99) of the sequences clustered within subgroup B1 and $51.5 \%(51 / 99)$ of the sequences clustered within subgroup B2.

\section{Temporal occurrence of HMPV in Kilifi}

During the 10-year period of surveillance described here, HMPV infections occurred in a seasonal pattern, mainly between the month of October of one year and April of the following year, Fig. 3, which was used to define an epidemic year, i.e. starting September of one year through August of the following year. Thus, subsequent analyses were done by epidemic year. Multiple subgroups co-circulated within a single epidemic year from 2007 to 2016, Fig. 4b. There was a shift in dominance from A2b (2007 to 2011) to B1 (2012 to 2014) with subsequent rise of the A2c in recent years. Notably, B2 subgroups remained persistent but non-dominant. A2b viruses circulated only until November 2012.

Table 1 Number of samples tested for HMPV and cases positives per year from 2007 to 16 amongst pneumonia paediatric admissions to Kilifi County Hospital, Kenya

\begin{tabular}{|c|c|c|c|c|c|}
\hline Year & Admissions $^{a}$ & Eligible $^{b}$ & Tested $^{c}$ & HMPV Positive & HMPV Prevalence (95\% Cl) \\
\hline 2007 & 3506 & 1174 & 726 & 21 & $2.90(1.80-4.39)$ \\
\hline 2008 & 3204 & 1009 & 435 & 34 & $7.80(5.47-10.75)$ \\
\hline 2009 & 3633 & 1105 & 526 & 46 & $8.70(6.47-11.49)$ \\
\hline 2010 & 3091 & 1090 & 903 & 27 & $3.00(1.98-4.32)$ \\
\hline 2011 & 3005 & 906 & 730 & 32 & $4.40(3.02-6.13)$ \\
\hline 2012 & 2715 & 863 & 943 & 50 & $5.30(3.96-6.93)$ \\
\hline 2013 & 2074 & 566 & 540 & 28 & $5.20(3.47-7.41)$ \\
\hline 2014 & 2947 & 797 & 695 & 18 & $2.60(1.54-4.06)$ \\
\hline 2015 & 3029 & 793 & 658 & 8 & $1.20(0.53-2.38)$ \\
\hline 2016 & 2803 & 776 & 600 & 10 & $1.70(0.80-3.04)$ \\
\hline Overall & 30,007 & 9079 & 6756 & 274 & 4.10 \\
\hline
\end{tabular}

Abbreviations: Cl Confidence intervals, HMPV Human metapneumovirus

a. Admissions under 5 years of age; b. Cases of severe or very severe pneumonia amongst the admissions [29]; c. Samples eligible for testing from the pneumonia admissions included only residents from within the Kilifi Health and Demographic Surveillance System (KHDSS) for years 2007-9, and residents or non-residents for other years 
Table 2 HMPV positives stratified by age of patients admitted to Kilifi County Hospital, Kenya 2007-2016

\begin{tabular}{lll}
\hline Age Categories & Frequency & Percent \\
\hline $0-2 \mathrm{M}$ & 51 & 18.61 \\
$3-5 \mathrm{M}$ & 67 & 24.45 \\
$6-8 \mathrm{M}$ & 43 & 15.69 \\
$9-11 \mathrm{M}$ & 35 & 12.77 \\
$12-17 \mathrm{M}$ & 20 & 7.30 \\
$18-23$ & 20 & 7.30 \\
$25-35 \mathrm{M}$ & 27 & 9.85 \\
$36+\mathrm{M}$ & 11 & 4.01 \\
Total & 274 & 100 \\
\hline
\end{tabular}

HMPV subgroup prevalence and dominance in Kilifi was compared to other countries around the world. This was based on analysis of $714 \mathrm{~F}$ gene sequences from 18 different countries and $500 \mathrm{G}$ gene sequences from 15 countries retrieved from GenBank (Additional file 2). General agreement in subgroup prevalence patterns was observed for Kilifi and global data sets, the predominance of A2b and B2 in 2007-2010 and subsequent rise of $\mathrm{B} 1$ and $\mathrm{A} 2 \mathrm{c}$ in years that followed, Fig. 4. Notably, at finer levels there were differences in subgroup temporal occurrence. Globally, clades A2b, A2c and subgroup B1 persisted over the 9 years. In contrast, in Kilifi only subgroup B2 persisted over the 9 years. The dominance of B1 strains in 2011 was also evident globally. A1 and A2a were the least commonly detected subgroups globally and not observed in Kilifi.

\section{Phylogenetic analysis of Kilifi HMPV viruses}

Based on maximum likelihood phylogenies of $F$ and $G$ genes, sequences from consecutive epidemics largely clustered together. However, across multiple epidemics sequences from the earlier years clustered together away from those in recent years, Figs. 5 and 6. Further analysis of $\mathrm{G}$ gene sequences aligned temporally, revealed circulation of multiple variants of a single subgroup in a single epidemic year (see Additional files 4 and 5). Each variant differed by at least five nucleotides (Additional

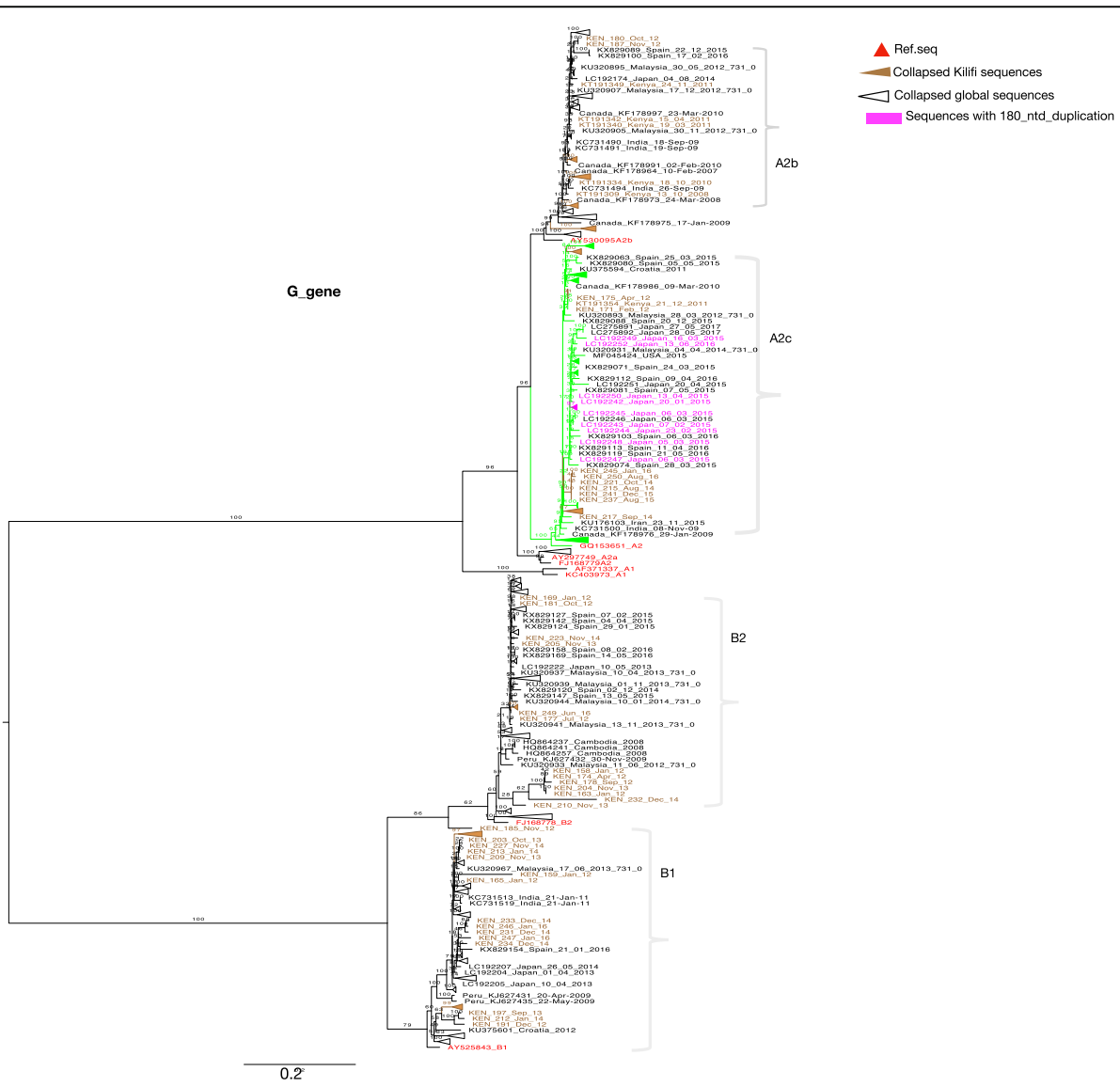

Fig. 1 A ML phylogenetic tree of 600 Gene sequences constructed using Kilifi sequences and sequences obtained from GenBank, collected from 2007 to 2016. Sequences were subtyped using references sequences retrieved from GenBank. Reference sequences colored in red. The numbers next to branches indicate the bootstrap values. Subgroups were confirmed if sequences clustered with the reference sequences within a major branch with $>70 \%$ bootstrap support. Taxa colored in magenta represent strains that have 180 nucleotide duplications retrieved from GenBank. Kilifi sequences are colored in brown 


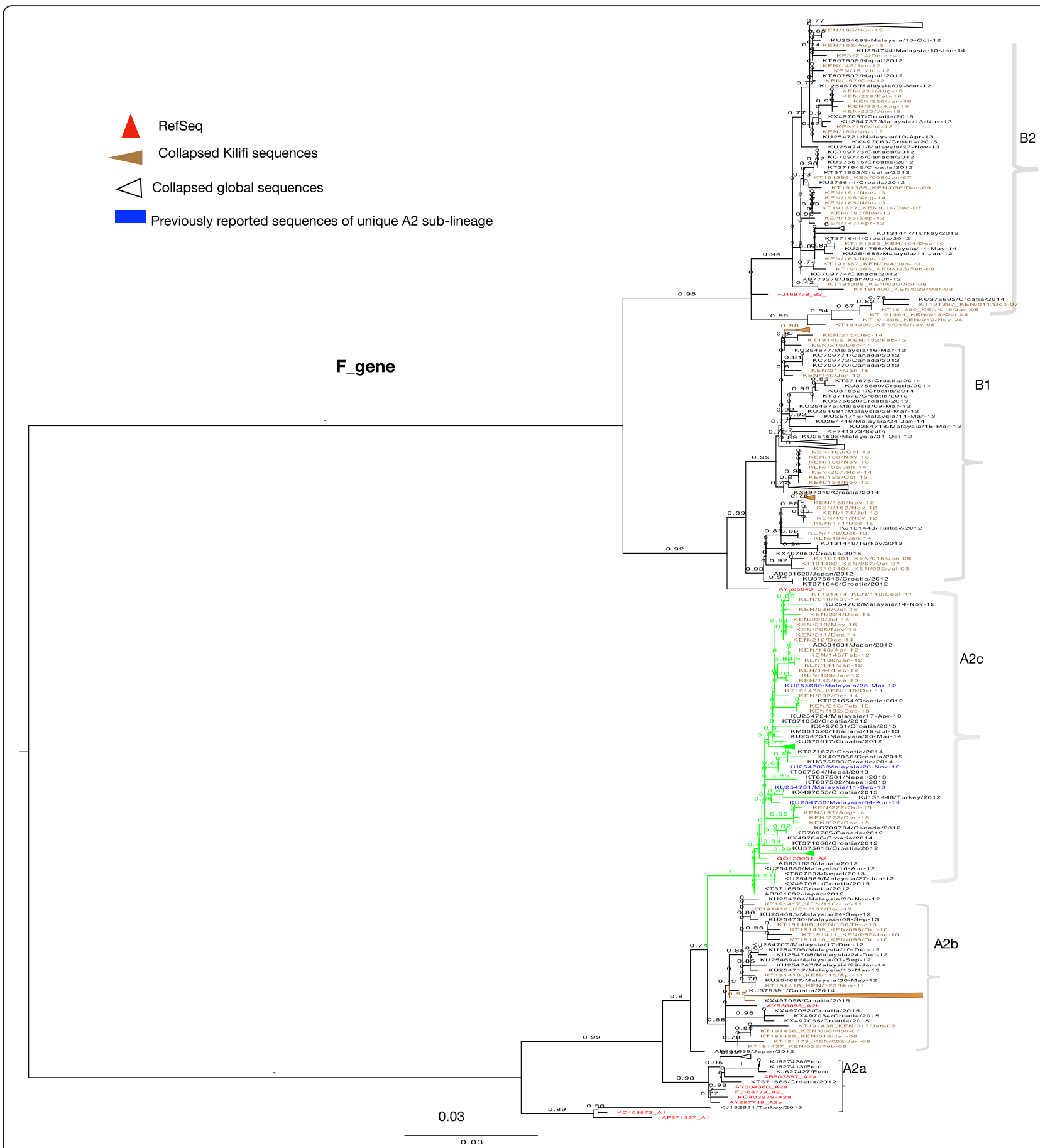

Fig. 2 A ML phylogenetic tree of 563 unique F Gene sequences constructed using Kilifi sequences and sequences obtained from GenBank, collected from 2007 to 2016. Sequences were subtyped using references sequences retrieved from GenBank. Reference sequences colored in red. The numbers next to branches indicate the bootstrap values. Subgroups were confirmed if sequences clustered with the reference sequences within a major branch with $>70 \%$ bootstrap support. Taxa colored in blue represent sequences of previously reported unique sub-lineage of A2, retrieved from GenBank. Kilifi sequences are colored in brown

files 4 and 5) and clustered into separate clades, Fig. 6. In comparison to contemporaneous viruses $(714 \mathrm{~F}$ gene sequences and $500 \mathrm{G}$ gene sequences) (Additional file 2), viruses from Kilifi and those from other locations were highly similar, with an average nucleotide identity of 97.1 and $95.6 \%$ (in F gene), and 90.4 and $85.9 \%$ (in G gene) for groups A and B, respectively. In phylogenies of $\mathrm{F}$ and G, combining contemporaneous and Kilifi 


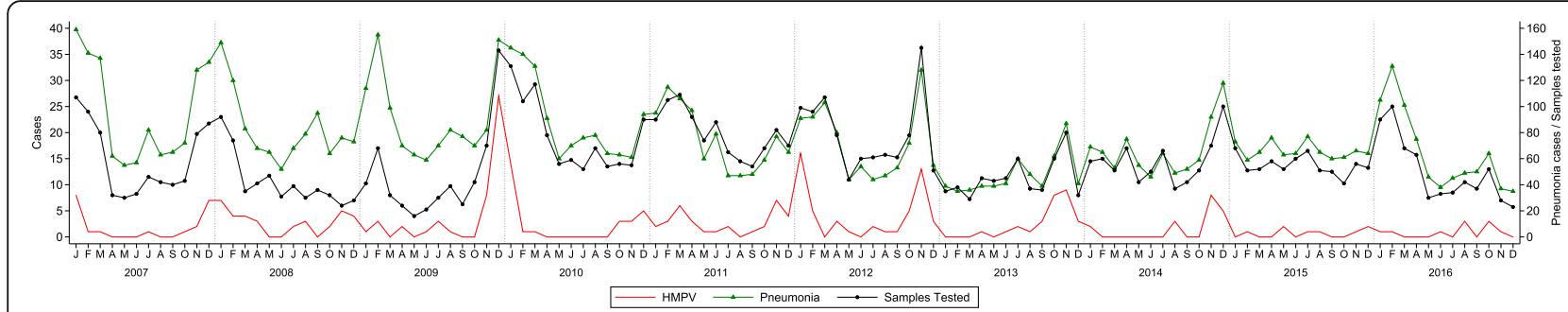

Fig. 3 Temporal distribution of HMPV cases identified monthly from surveillance of severe and very severe pneumonia cases aged under 5 years admitted to Kilifi County Hospital, Kenya 2007-2016. Number pneumonia cases and number tested are shown on the secondary Y axis and HMPV cases detected shown on the primary $Y$ axis. Samples eligible for testing from the pneumonia admissions included only residents from within the Kilifi Health and Demographic Surveillance System (KHDSS) for years 2007-9, and residents or non-residents for other years

sequence data, the Kilifi sequences did not cluster separately from others but interspersed with sequences from Canada, Spain, Malaysia, Japan, USA, India and Croatia (Figs. 1 and 2), and similarly, those viruses collected from earlier years in Kilifi clustered away from those in recent years.

\section{F gene sequence analysis}

Due to partial gene sequencing ( $345 \mathrm{bp}$ ) of the previously reported $\mathrm{F}$ gene Kilifi sequences retrieved from GenBank [28], combined Kilifi sequences were trimmed to $345 \mathrm{bp}$. The $\mathrm{F}$ gene sequences were highly conserved both at the nucleotide (nt) and amino acid (aa) level with

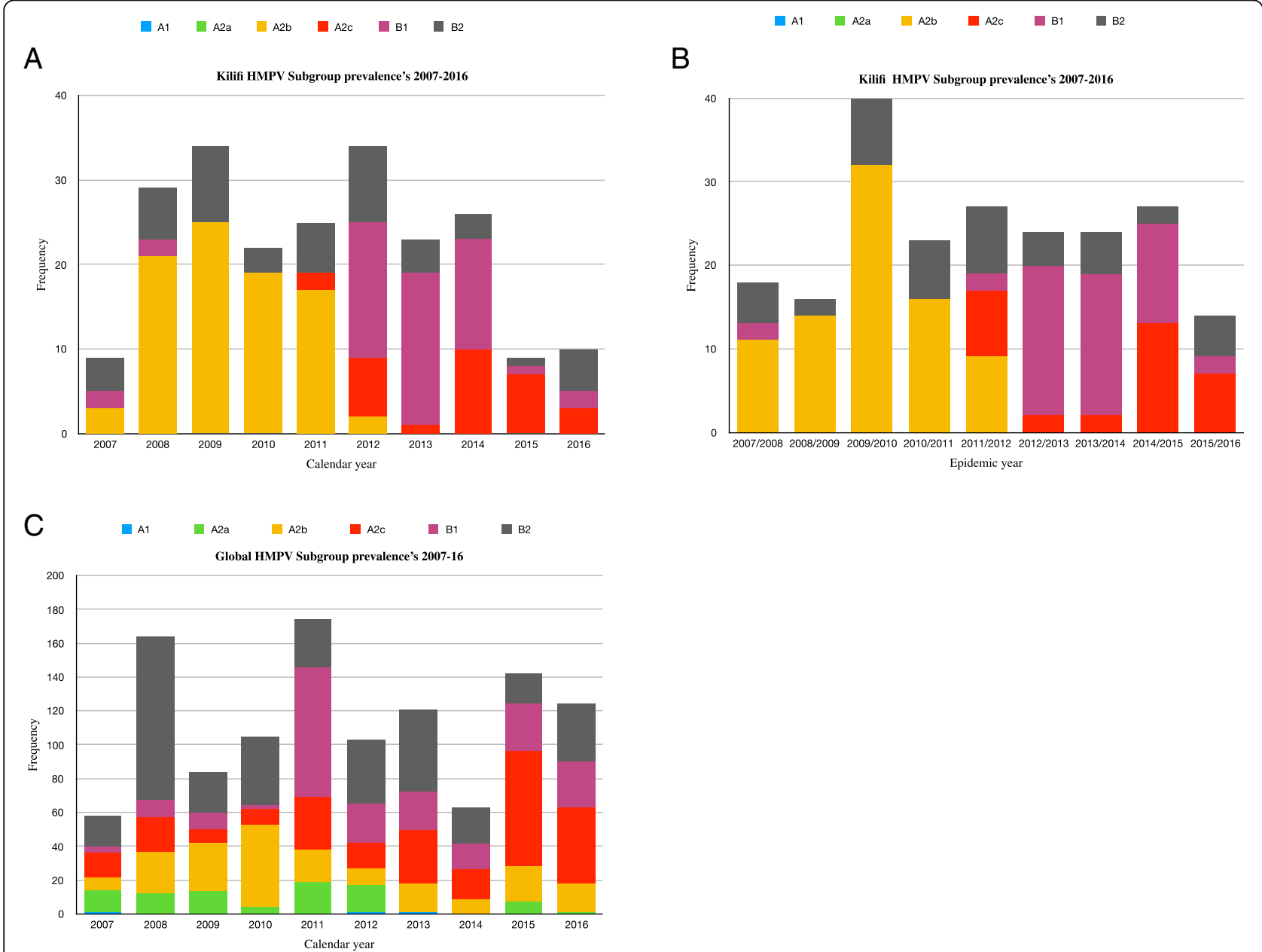

Fig. 4 HMPV subgroup prevalence patterns Kilifi versus Global from 2007 to 2016. Panel a, Kilifi subgroup temporal patterns derived from Kilifi sequence data determined by calendar year. Panel b, Kilifi subgroup temporal patterns determined by epidemic year. Panel c, global subgroup temporal patterns determined from combined $714 \mathrm{~F}$ sequences and $500 \mathrm{G}$ sequences retrieved from GenBank 


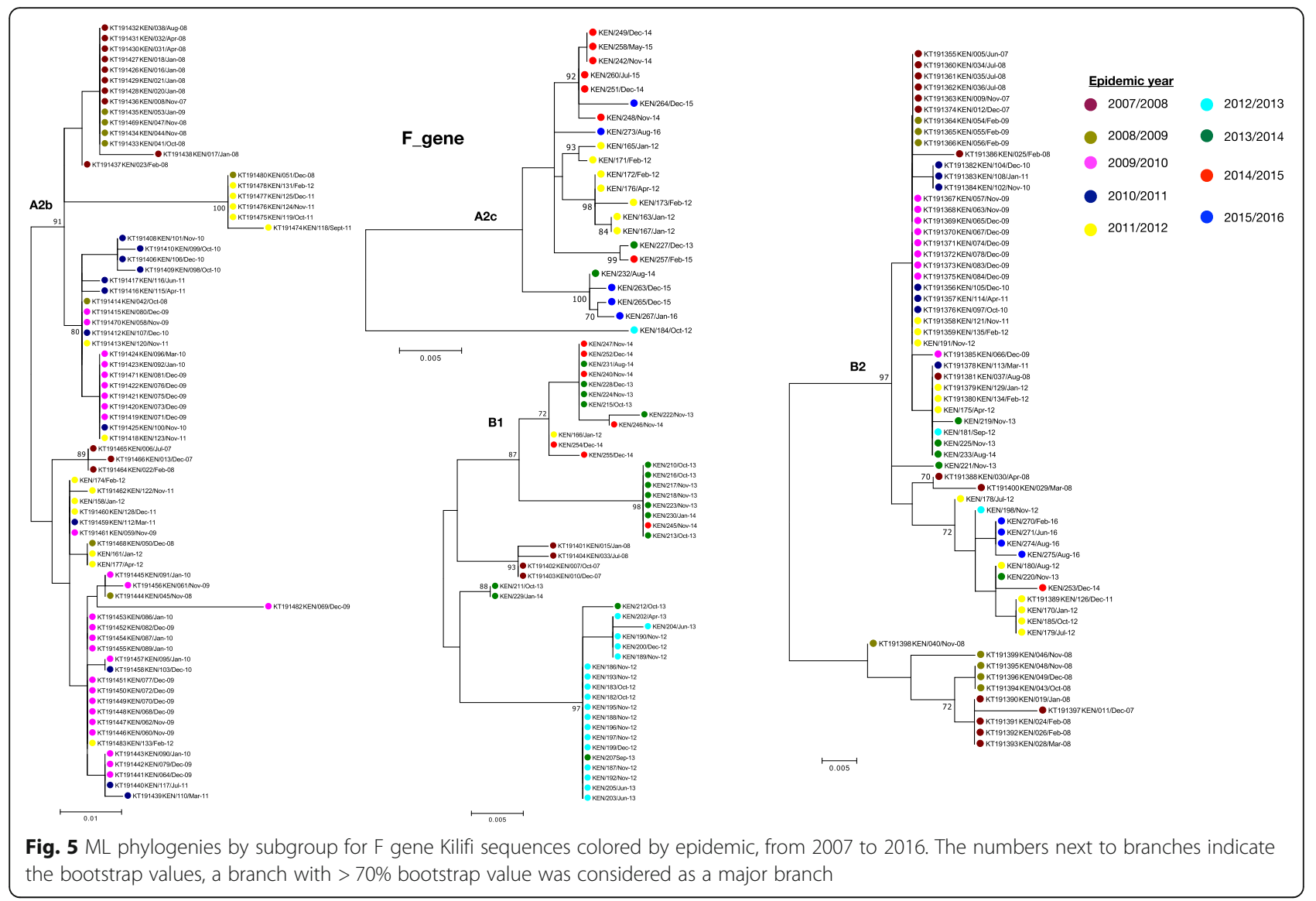

overall mean sequence identity of $89 \%$ (nt) and $97 \%$ (aa). Within the individual subgroups, the nucleotide and amino acid sequences were more conserved: there was $100 \%$ aa sequence similarity within clades A2b, A2c and subgroup B1, and $99.9 \%$ within B2 subgroup, and the mean nucleotide identities were $98.2 \%$ within B1, 99.2\% within A2c, 98.3\% within both A2b and B2. The evolutionary rates for $\mathrm{F}$ gene were estimated at $1.220 \times \mathbf{1 0}^{-3}$ $\left(95 \%\right.$ highest posterior density $(\mathrm{HPD})=1.317 \times 10^{-3}$ to $\left.5.518 \times 10^{-3}\right), \mathbf{1 . 8 5 7 \times 1 0 ^ { - 3 }}(95 \% \mathrm{HPD}=1.111 \times 10-3$ to $\left.2.289 \times 10^{-3}\right), \quad \mathbf{1 . 2 4 2 \times 1 0 ^ { - 3 }}\left(95 \% \mathrm{HPD}=8.303 \times 10^{-4}\right.$ to $\left.1.689 \times 10^{-3}\right)$ and $\mathbf{1 . 0 5 0} \times \mathbf{1 0}^{-\mathbf{3}}\left(95 \% \mathrm{HPD}=5.937 \times 10^{-4}\right.$ to $\left.1.600 \times 10^{-3}\right)$, nucleotide substitutions/site/year for $\mathrm{A} 2 \mathrm{c}, \mathrm{A} 2 \mathrm{~b}, \mathrm{~B} 1$ and B2, respectively. Selection pressure analysis of the $F$ gene showed overall mean $\mathrm{dN} / \mathrm{dS}$ ratio of $\omega=0.10, \omega=0.15, \omega=0.01$ and $\omega=0.12$ for $\mathrm{A} 2 \mathrm{c}, \mathrm{A} 2 \mathrm{~b}, \mathrm{~B} 1$ and B2, respectively (see Additional file 6). Using M2a and M3 models, no codon sites were identified as positively selected among the four types (sub-lineage A2b, sub-lineage A2c, B1 and B2 subgroups). (Additional file 6).

\section{G gene sequence analysis (Kilifi)}

$\mathrm{G}$ gene sequences were less conserved compared to $\mathrm{F}$ gene sequences with overall mean sequence identity of
73\% (nt) and 56\% (aa). However, within subgroups the sequences were highly conserved: the mean identities were estimated at 97\% (nt) and 96\% (aa) for clade A2c, 93\% (nt) and 89\% (aa) for clade A2b, 94\% (nt) and 90\% (aa) for B1, 92\%(nt) and $87 \%$ for B2 subgroups. The deduced $G$ gene amino acid sequences differed in lengths among subgroups: clade A2c (220aa), clade A2b (218 and 229aa), B2 (238aa) and B1 (232 and 242aa) long. The varying protein lengths for clade $\mathrm{A} 2 \mathrm{~b}$ and $\mathrm{B} 1$ subgroups were due to changes in stop codon positions. The evolutionary rates for the $\mathrm{G}$ gene were estimated at $3.420 \times \mathbf{1 0}^{-3}$ $\left(95 \%\right.$ HPD interval $=1.317 \times 10^{-3}$ to $\left.5.518 \times 10^{-3}\right)$, $\mathbf{8 . 0 2 2} \times \mathbf{1 0}^{-3}\left(95 \% \mathrm{HPD}=5.893 \times 10^{-3}\right.$ to $\left.1.060 \times 10^{-2}\right)$ and $9.570 \times 10^{-3}$ (95\% HPD $=5.911 \times 10^{-3}$ to $1.33 \times$ $10^{-2}$ ) nucleotide substitutions/site/year for A2c, A2b and $\mathrm{B} 1$, respectively. Evolutionary rate estimates for subgroup B2 viruses were not determined, effective sample size (ESS) for all parameters failed to reach the cut-off (200) required for confirmation of convergence in BEAST analysis. Among all the subgroups, A2c exhibited significantly lower substitution rates within the $\mathrm{G}$ gene $\left(95 \% \mathrm{HPD}=1.317 \times 10^{-3}\right.$ to $5.518 \times$ $\left.10^{-3}\right)$. The estimated times of the most common ancestor of the individual subgroups dated to recent 


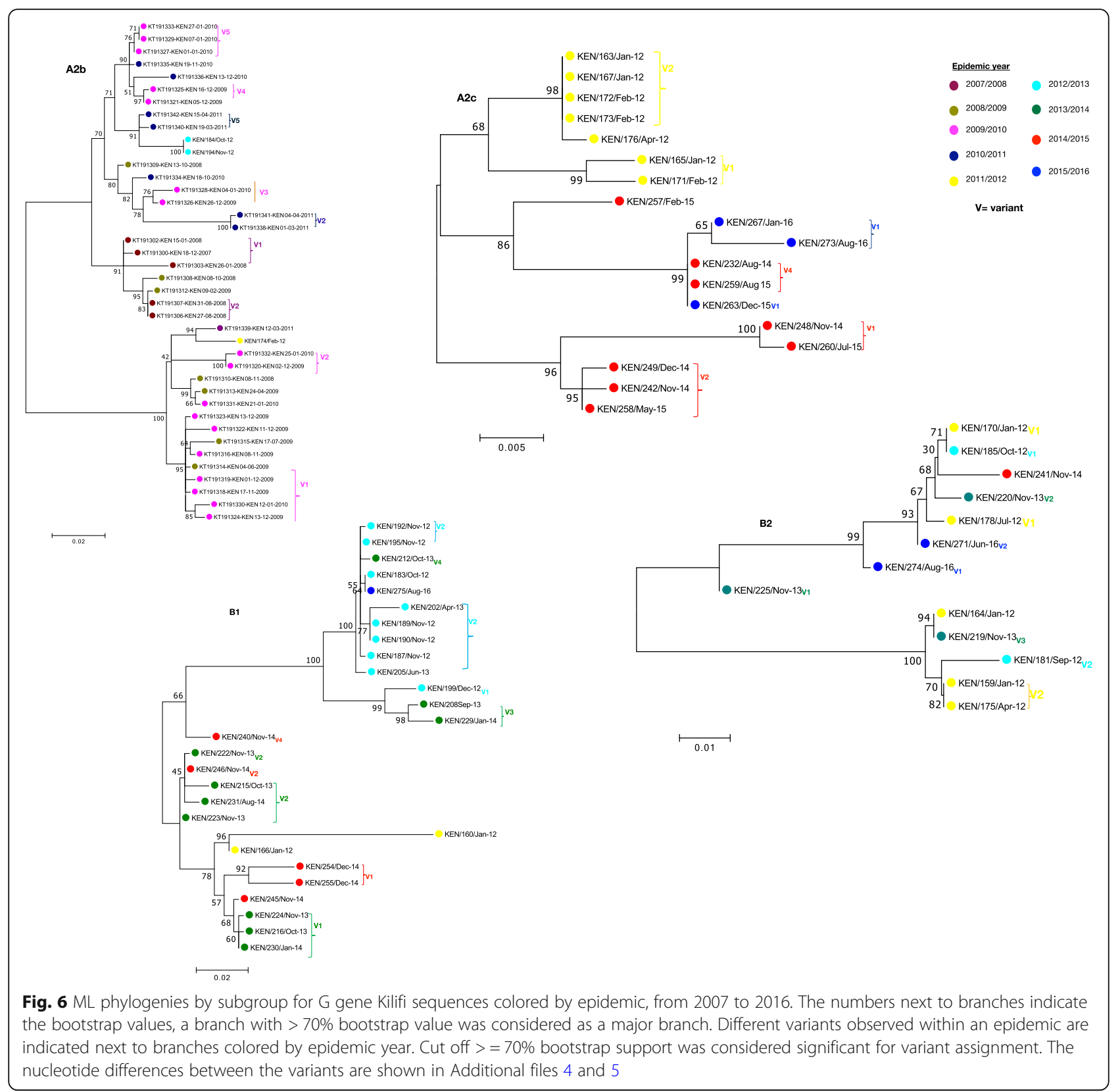

origin in both $\mathrm{F}$ and $\mathrm{G}$ genes, i.e., 2009 (95\% HPD = 2005-2011) for clade A2c sequences, 2008 (95\% $\mathrm{HPD}=2006-2009)$ for clade A2b, 2005 (95\% HPD = 2002-2007) for B1 and 2001(95\% HPD = 1997-2005) for $\mathrm{B} 2$ sequences. The estimated mean $\mathrm{dN} / \mathrm{dS}$ ratio ( $\omega)$ was $<1$ for A2b $(\omega=0.57)$, A2c $(\omega=0.38)$ and $\mathrm{B} 1(\omega=0.78)$. For the subgroup $\mathrm{B} 2$, a higher average $\mathrm{dN} / \mathrm{dS}$ ratio $(\omega=1.21)$ was determined for $\mathrm{G}$ gene, indicating a signal of positive selection (Additional file 6). Using M2a and M3 models, 3 positively selected sites were identified within A2c (17, 24, 28, and) and A2b (87, 123 and 135), 1 site (96) in B1 and 2 sites $(12,127)$ within B2 sequences (Additional file 6).

\section{HMPV subgroup and disease severity}

We did not observe a statistically significant association between HMPV subgroup and pneumonia severity ( $p$ value $=0.264)($ Table 3$)$.

\section{Discussion}

There remains limited information on long-term circulation patterns and prevalence of HMPV in Africa. This study reports HMPV subtype incidence and molecular evolution in coastal Kenya over a 10 -year period of surveillance (2007 to 2016). HMPV incidence in children under 5 years of age varied annually ranging from 1.2 to $8.7 \%$ and $71.5 \%$ of HMPV infections were in children 
Table 3 Association between different HMPV subgroups and disease severity

\begin{tabular}{|c|c|c|c|c|}
\hline Subgroup & & WHO Pneumonia Status & & Total \\
\hline & & Severe & Very severe & \\
\hline \multirow[t]{2}{*}{$A 2 b$} & $n$ & 71 & 13 & 84 \\
\hline & $\%$ & 84.52 & 15.48 & 100 \\
\hline \multirow[t]{2}{*}{$\mathrm{A} 2 \mathrm{C}$} & $\mathrm{n}$ & 20 & 2 & 22 \\
\hline & $\%$ & 90.91 & 9.09 & 100 \\
\hline \multirow[t]{2}{*}{ B1 } & $\mathrm{n}$ & 35 & 13 & 48 \\
\hline & $\%$ & 72.92 & 27.08 & 100 \\
\hline \multirow[t]{3}{*}{ B2 } & $n$ & 41 & 10 & 51 \\
\hline & $\%$ & 80.39 & 19.61 & 100 \\
\hline & $n$ & & & \\
\hline \multirow[t]{3}{*}{ Total } & $\%$ & 167 & 38 & 205 \\
\hline & & 81.46 & 18.54 & 100 \\
\hline & & & & $p$-value $=0.264$ \\
\hline
\end{tabular}

aged $<2$ years. Wide variation in annual prevalence has been reported elsewhere from long-term studies of acute respiratory infection (ARI) aetiology in children: in Germany, HMPV prevalence over 10 years of surveillance varied between 1.4 and $32.8 \%$ [21], and in Italy, the prevalence fluctuated from 37 to $7 \%$ and then to $43 \%$ over a 3 year period [24]. To date the cause of the varied prevalence and whether there is difference in disease severity among HMPV subgroups remains unclear. Different studies report contrasting results on the association of disease severity among the HMPV subgroups $[10,40]$. In this study, no significant association was found between subgroup and disease severity or clinical presentation. Further studies are necessary to evaluate whether changes in subgroup are associated with changes in disease severity and prevalence. Climatic factors such as temperature, rainfall and relative humidity have also been shown to influence HMPV activity in the tropics [23]. In this study, seasonal increase in cases tended to coincide with low rainfall, lower relative humidity and higher temperatures. Further investigation is required to determine whether changes in such environmental factors influence HMPV activity.

Kilifi HMPV subgroup prevalence patterns partly mirrored global patterns, there was predominance of $\mathrm{A} 2 \mathrm{~b}$ and B2 in 2007-2010 and subsequent rise of B1 and A2c in years that followed. B2 viruses persisted over the 9 years. A1 and A2a were the least commonly detected subgroups globally and this could probably explain why they were not detected in Kilifi. In contrast to continued circulation of clade A2b [18], in Kilifi, A2b circulated from 2007 to November 2012 and no longer. Occurrence of the clade A2c [41] has increased in Kilifi in recent years, as similarly reported in Bangladesh and
Croatia [15, 16], suggestive of global spread of HMPV variants. Based on F and $G$ phylogenies, some of previously reported sequences from Europe, Asia and USA that either had not been sub-grouped or assigned to clade A2b, clustered within A2c, consistent with findings from a previous report [15]. Similarly, previously reported sequences of the unique A2 clade reported in Malaysia [23] also clustered in this clade (A2c). Recently, two novel HMPV A2 strains with 180 or 111 nucleotide duplication in the $G$ gene have also been reported [17, 20]. These strains were not observed in Kilifi. In the phylogenetic trees they clustered within A2c together with other A2c sequences without duplication including Kilifi A2c strains. Our findings reaffirm the circulation of A2c clade and point out the need for proper methods for classification of HMPV lineages. The replacement patterns of HMPV subgroups could be attributed to subgroup-specific herd immunity offering protection against circulating homologous strains but not against heterologous strains. This observation is not unique to Kilifi, as similar patterns were reported from long-term surveillance studies in Germany and France [21, 42]. Consistent with earlier studies, $G$ gene exhibited higher rates of evolution compared to $\mathrm{F}$ gene [2]. $\mathrm{F}$ gene is more conserved and our findings concur with previous studies on $\mathrm{F}$ protein diversity [43]. The rates of $\mathrm{G}$ gene evolution were in the range of $3.42 \times 10^{-3}$ to $9.57 \times 10^{-3}$, similar to findings from previous studies $[44,45]$. Among the subgroups, the rates of evolution for $\mathrm{A} 2 \mathrm{~b}$ and $\mathrm{B} 1$ were at least 2 fold higher than other subgroups, this concurs with the findings from a similar study [44]. In general, higher polymorphism has been reported in HMPV G gene and further within the subgroups resulting from events such as changes in alternative transcription termination codons and insertions [46]. This could explain the differences in evolution rates. Further investigations are required to give more insights on the differences in evolution rates and its implication. Compared to other Pneumoviruses, such as human respiratory syncytial virus (HRSV), HMPV G gene has been shown to have higher rates of evolution [45]. Our estimates show comparable results, the rates for HMPV A2b and B1 were 3 to 4 times higher than those reported for HRSV $(3.58 \times$ $10^{-3}, 95 \% \mathrm{HPD}=3.04 \times 10^{-3}$ to $4.16 \times 10^{-3}$ ) [47]. The difference in the rates of evolution can be linked to strong association between neutralizing epitopes and positively selected sites reported for RSV G gene [48]. In contrast to the case for other paramyxoviruses, such as $\mathrm{RSV}$, the HMPV G protein is not a major neutralizing or protective antigen [49].

Temporal analysis revealed circulation of multiple subgroups within a single epidemic. Further analysis of the G glycoprotein encoding gene revealed circulation of multiple variants for a single subgroup within an epidemic. 
Multiple variants observed might be attributed to high rate of $\mathrm{G}$ gene evolution (substitutions/site/year) resulting in diversification in situ during a season. However, the level of variation observed within a subgroup within epidemics was greater that would be expected from in situ diversification, implying multiple variant introductions of a subgroup during single epidemic years.

As is the norm for HMPV detection in respiratory samples, we used molecular PCR-based diagnostics [6]. Recent studies have shown that mutations at primer and probe binding sites can lead to false negative diagnostic results and hence underestimation of disease burden [50, 51]. Evaluation of the in-house diagnostic rRT-PCR assay would be important to determine whether there are any missed variants/subgroups and whether this can be associated with the apparent gradual decline in HMPV prevalence observed in the current dataset. There is also some evidence that reduction in bacterial pneumonia (e.g. S. pneumoniae), as has been seen in this coastal location over this study period [52], results in a reduction in viral pneumonia $[53,54]$. Future investigations will be necessary to give more insight.

The analysed Kilifi F and G protein encoding genes were generally determined to be under purifying selection pressure, which drives RNA virus evolution by purifying the deleterious mutations due to RNA replication errors [55]. However, for the B2 subgroup, a higher dN/ dS ratio was observed in $G$ gene sequences suggestive of diversifying selection within $\mathrm{B} 2$ viruses. The distinct diversifying selection and persistence of the $\mathrm{B} 2$ viruses observed requires further investigation. In this study, our $\mathrm{F}$ and $G$ genes sequence analyses were based on partial gene lengths, $345 \mathrm{bp}$ and $640 \mathrm{bp}$, respectively. Therefore, our results on the genetic distance estimates, evolutionary and selection pressure analysis should be interpreted with caution. The partial lengths may have reduced our potential to discriminate genetic clusters, a possible explanation for higher sequence similarity observed between and within the subgroups for $\mathrm{F}$ gene. Overall, although our sequence analyses were limited to partial lengths, the newly designed $F$ and $G$ genes primers allowed full length sequencing of the two genes for newly generated data. In addition, the newly designed G gene subtype specific primers allowed sequencing of all HMPV subtypes and significantly improved G PCR recovery by two-fold compared to previously reported assay. This improved the study power to characterise the different circulating HMPV variants. Overall, we successfully collected clinical samples from $74 \%$ (6756/ 9076) of the study enrolled participants and characterised 75\% (205/274) of the HMPV viruses identified. In this study we failed to collect samples from $24 \%$ of the eligibles. We have previously reported similar results, which results from refusals and difficulty in collection of nasal specimens from children with very severe disease [29]. Hence, if some HMPV variants are associated with disease severity there may be bias in the prevalence and variant composition estimates. However, the proportion not collected shows no systematic change over time. In addition, the proportion of very severe pneumonia cases was high among sampled eligibles compared to unsampled eligibles. Therefore, it's unlikely there was any bias in the prevalence estimates of HMPV groups or subgroups.

Our analyses on HMPV epidemiology in Kilifi were limited to two viral genes only. Whole genome sequencing might give more insights into transmission, HMPV subgroup characterisation and molecular evolution. Our estimation and inference on the association between HMPV subgroup and disease severity was biased to inpatient surveillance data, and therefore future studies should include outpatient surveillance data. In addition, inpatient surveillance and sampling of HMPV infections might not be representative of the full variant population circulating in this community and not correctly reflect incidence and prevalence, as HMPV infections have also been reported in outpatient settings [56]. Future studies across different locations in Kenya and in Africa will be important for tracing the introduction and transmission patterns of the virus.

\section{Conclusions}

In conclusion, this report shows HMPV activity characterised by marked annual variation in occurrence, and in subgroup prevalence patterns (reflecting those in other locations globally,) gradual replacement of subgroups over time, and multiple circulating variants each epidemic varying year to year. This suggests, widescale and rapid circulation of HMPV subgroups, seasonal epidemics resulting from multiple introductions rather than single source, and possible evidence of herd immunity replacement at the subgroup and within-subgroup cluster level.

\section{Additional files}

Additional file 1: Newly designed $F$ and $G$ gene PCR and sequencing primers. (DOCX $15 \mathrm{~kb}$ )

Additional file 2: Panel A and B, GenBank accession numbers for global contemporaneous $F(714)$ and $G(500)$ gene sequences retrieved from GenBank. Panel C, GenBank accession numbers for Kilifi sequences detected by alternative rt-PCR assay. (CSV $11 \mathrm{~kb}$ )

Additional file 3: Phylogenetic analysis of Kilifi sequences and sequences retrieved from GenBank constructed using only full F gene sequences (1593 bp) to further asses the clustering of HMPV subgroups. A total 185 full F sequences were used. Sequences were subtyped using references sequences retrieved from GenBank. Reference sequences colored in red. The numbers next to branches indicate the bootstrap values, a branch with $>70 \%$ bootstrap value was considered as a major branch. (PDF $44 \mathrm{~kb}$ ) 
Additional file 4: Changes in $\mathrm{G}$ gene nucleotide sequences for sequences collected from Kilifi. Sequences were aligned by subgroup and by epidemic year for Clade A2b and A2c. Nucleotide differences were determined and indicated by vertical colored bars. Orange red represent Adenine (A), Crimson represent Thymine (T), Indigo represents Guanine (G) and slate blue represent Cytosine (C) nucleotide. (PDF $261 \mathrm{~kb}$ )

Additional file 5: Changes in $\mathrm{G}$ gene nucleotide sequences for sequences collected from Kilifi. Sequences were aligned by subgroup and by epidemic year for subgroups B1 and B2. Nucleotide differences were determined and indicated by vertical colored bars. Orange red represent Adenine (A), Crimson represent Thymine (T), Indigo represents Guanine (G) and slate blue represent Cytosine (C) nucleotide. (PDF 167 kb)

Additional file 6: Parameter estimates, dN/dS ratios, positively selected sites and log likelihood scores for $F$ and $G$ gene nucleotide sequences for the HMPV subgroups identified between 2007 and 2016. (PDF 48 kb)

\section{Abbreviations}

ARI: Acute respiratory infections; HMPV: Human metapneumovirus; HPD: Highest posterior density; KCH: Kilifi County Hospital; KHDSS: Kilifi Health and Demographic Surveillance System; RNA: Ribonucleic acid; rRTPCR: Real time Reverse Transcription Polymerase Chain Reaction; tMRCA: Time to the most recent common ancestor

\section{Acknowledgements}

We thank the study participants for providing the study samples. We thank all members of the Virus Epidemiology and Control (VEC) Research Group in Kilifi who were involved at various stages of this study that has allowed this current report to be written. We thank Dr. Matt Cotten for sharing his scripts for generating the hiliter plots. We also thank the parents and guardians of the children for accepting to participate in this study. This work is published with permission of director KEMRI.

\section{Authors' contributions}

DJN and CNA: conceived and designed the study, supervised the work and gave technical guidance. JOW, JRO, EK: participated in laboratory, sequence and Phylogenetic analyses, GPO: carried clinical data analysis and data curation. JOW, DJN and CAN: wrote the manuscript. All authors read and approved the final manuscript

\section{Funding}

This study was supported by the Wellcome Trust, United Kingdom (grants 102975, 100542, 084633, and 077092). The funder had no role in other aspects of the study including its design, data collection, data analysis, data interpretation, or writing of this manuscript.

\section{Availability of data and materials}

All data generated or analysed during this study has been deposited to the Virus Epidemiology and Control (VEC), KEMRI-Wellcome Trust Research Programme, data server under the DOI: https://dataverse.harvard.edu/dataset. xhtml?persistentld=doi:10.7910/DVN/MJPRLV.

\section{Ethics approval and consent to participate}

The Kenya Medical Research Institute Scientific and Ethics Review Unit (SERU) approved the study. A written informed consent was obtained from participant's parent or guardian.

\section{Consent for publication}

$$
\text { Not applicable. }
$$

\section{Competing interests}

The authors declare that they have no competing interest.

\section{Author details}

${ }^{1}$ Kenya Medical Research Institute (KEMRI) -Wellcome Trust Research Programme, Kilifi, KEMRI Centre for Geographic Medicine Research - Coast, Kilifi, Kenya. ${ }^{2}$ School of Health and Human Sciences, Pwani University, Kilifi, Kenya. ${ }^{3}$ School of Life Sciences, and Zeeman Institute for Systems Biology and Infectious Disease Epidemiology Research (SBIDER), University of Warwick, Coventry, UK.
Received: 29 March 2019 Accepted: 15 August 2019

Published online: 30 August 2019

\section{References}

1. Schildgen V, van den Hoogen B, Fouchier R, Tripp RA, Alvarez R, Manoha C, et al. Human metapneumovirus: lessons learned over the first decade. Clin Microbiol Rev. 2011:24(4):734-54.

2. II KJ, Park S, Lee I, Park KS, Kwak EJ, Moon KM, et al. Genome-wide analysis of human metapneumovirus evolution. PLoS One. 2016;11(4):1-19.

3. Edwards Kathryn M, Yuwei Z, Griffin Marie R, Weinberg Geoffrey A, Hall Caroline B, Szilagyi Peter G, Staat Mary A, PMM IM, WJ V. Burden of human Metapneumovirus infection in young children. N Engl J Med. 2013;368(7): 633-43.

4. Neto DF de L, Carraro E, Hern CF, Granato es, Bellei NCJ. Human metapneumovirus infections in adults associated with respiratory illness in Sao Paulo, Brazil. Journal of Public Health and Epidemioly 2013;5(6):257-261.

5. van den Hoogen BG, de Jong JC, Groen J, Kuiken T, de Groot R, Fouchier RA, et al. A newly discovered human pneumovirus isolated from young children with respiratory tract disease. Nat Med. 2001;7(6):719-24.

6. Panda S, Mohakud NK, Pena L, Kumar S. Human metapneumovirus: review of an important respiratory pathogen. International Journal of Infectious Disease . 2014;25:45-52

7. Shafagati $\mathrm{N}$ and Williams J. Human metapneumovirus - what we know now [version 1; peer review: 2 approved]. F1000Research 2018, 7(F1000 Faculty Rev):135 (https://doi.org/10.12688/f1000research.12625.1).

8. Van den Hoogen BG, van Doornum GJ, Fockens JC, Cornelissen JJ, Beyer $W E$, de Groot $R$, et al. Prevalence and clinical symptoms of human metapneumovirus infection in hospitalized patients. Journal of Infectious Disease. 2003;188(10):1571-7.

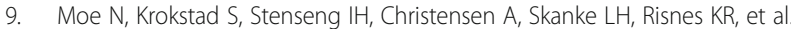
Comparing human metapneumovirus and respiratory syncytial virus: viral codetections, genotypes and risk factors for severe disease. PLoS One. 2017; 12(1):1-19.

10. Papenburg J, Hamelin M-È, Ouhoummane N, Carbonneau J, Ouakki M, Raymond F, et al. Comparison of risk factors for human Metapneumovirus and respiratory syncytial virus disease severity in young children. Journal of Infectious Disease. 2012;206(2):178-89.

11. Van Den Hoogen BG, Herfst S, Sprong L, Cane PA, Forleo-neto E, De Swart $R L$, et al. Antigenic and genetic variability of human Metapneumoviruses. Emerg Infect Dis. 2004;10(4):658.

12. Endo R, Ebihara T, Ishiguro N, Teramoto S, Ariga T, Sakata C, et al. Detection of four genetic subgroup-specific antibodies to human metapneumovirus attachment (G) protein in human serum. J Gen Virol. 2008:89(8):1970-7.

13. Biacchesi S, Skiadopoulos MH, Boivin G, Hanson CT, Murphy BR, Collins PL, et al. Genetic diversity between human metapneumovirus subgroups. Virology. 2003;315(1):1-9.

14. Huck B, Scharf G, Neumann-Haefelin D, Puppe W, Weigl J, Falcone V. Novel human metapneumovirus sublineage. Emerg Infect Dis. 2006;12(1):147-50.

15. Jagušić M, Slović A, Ljubin-Sternak S, Mlinarić-Galinović G, Forčić D. Genetic diversity of human metapneumovirus in hospitalized children with acute respiratory infections in Croatia. J Med Virol. 2017;89(11):1885-93.

16. Rahman MZ, Sumiya M, Sahabuddin M, Pell LG, Gubbay JB, Rahman R, et al. Genetic characterization of human metapneumovirus identified through community and facility-based surveillance of infants in Dhaka. Bangladesh Journal of Medical Virology. 2019;91(4):549-54.

17. Saikusa M, Kawakami C, Nao N, Takeda M, Usuku S, Sasao T, Nishimoto K, Toyozawa T. 180-nucleotide duplication in the $\mathrm{G}$ gene of human metapneumovirus A2b subgroup strains circulating in Yokohama City, Japan, since 2014. Front Microbiol. 2017:8:402.

18. Piñana M, Vila J, Gimferrer L, Valls M, Andrés C, Codina MG, et al. Novel human metapneumovirus with a 180-nucleotide duplication in the $\mathrm{G}$ gene. Future Microbiol. 2017;12(7):565-71.

19. Saikusa M, Nao N, Kawakami C, Usuku S, Sasao T, Toyozawa T, Takeda M, Okubo I. A novel 111-nucleotide duplication in the $\mathrm{G}$ gene of human metapneumovirus. Microbiol Immunol. 2017 Nov;61(11):507-12.

20. Saikusa M, Nao N, Kawakami C, Usuku S, Tanaka N, Tahara M, Takeda M, Okubo I. Predominant detection of the subgroup A2b human metapneumovirus strain with 111-nucleotide duplication in Yokohama City, Japan in 2018. Japanese journal of infectious diseases. 2019. (https://doi. org/10.7883/yoken.JJID.2019.124). 
21. Reiche J, Jacobsen S, Neubauer K, Hafemann S, Nitsche A, Milde J, et al. Human metapneumovirus: Insights from a ten-year molecular and epidemiological analysis in Germany. PLoS One. 2014;9(2):e88342.

22. Lo PA, Cammarota R, Apostoli P, Cella E, Fiorentini S, Babakir-Mina M, et al. Genetic variability and circulation pattern of human metapneumovirus isolated in Italy over five epidemic seasons. New Microbiology. 2011;34(4): 337-44.

23. Chow WZ, Chan YF, Oong XY, Ng LJ, Nor'E SS, Ng KT, et al. Genetic diversity, seasonality and transmission network of human metapneumovirus: identification of a unique sub-lineage of the fusion and attachment genes. Sci Rep. 2016;6(9):27730

24. Maggi F, Pifferi M, Vatteroni M, Fornai C, Tempestini E, Anzilotti S, et al Human metapneumovirus associated with respiratory tract infections in a 3year study of nasal swabs from infants in Italy. J Clin Microbiol. 2003;41(7): 2987-91.

25. Rafiefard F, Yun ZB, Örvell C. Epidemiologic characteristics and seasonal distribution of human metapneumovirus infections in five epidemic seasons in Stockholm, Sweden, 2002-2006. J Med Virol. 2008;80(9):161-8.

26. Green RJ, Kolberg JM. Neonatal pneumonia in sub-Saharan Africa. Pneumonia . 2016;0-1.

27. Liu L, Johnson HL, Cousens S, Perin J, Scott S, Lawn JE, et al. Global, regional, and national causes of child mortality: an updated systematic analysis for 2010 with time trends since 2000. Lancet. 2012;379(9832):2151-61.

28. Owor BE, Masankwa GN, Mwango LC, Njeru RW, Agoti CN, Nokes DJ. Human metapneumovirus epidemiological and evolutionary patterns in coastal Kenya, 2007-11. BMC Infect Dis. 2016;16(1):301.

29. Nokes DJ, Ngama M, Bett A, Abwao J, Munywoki P, English M, et al. Incidence and severity of respiratory syncytial virus pneumonia in rura Kenyan children identified through hospital surveillance. Clin Infect Dis. 2009:49(9):1341-9.

30. Scott JAG, Bauni E, Moisi JC, Ojal J, Gatakaa H, Nyundo C, et al. Profile : The Kilifi Health and Demographic Surveillance System ( KHDSS ).International Journal of Epidemiology 2012;(April): 41(3):650-7.

31. Berkley JA, Munywoki $P$, Ngama M, Abwao J, Bett A, Lassauniére $R$, et al Viral etiology of severe pneumonia among Kenyan young infants and children. Jama. 2010;303(20):2051-7.

32. Hammitt LL, Kazungu S, Welch S, Bett A, Onyango CO, Gunson RN, et al. Added value of an oropharyngeal swab in detection of viruses in children hospitalized with lower respiratory tract infection. J Clin Microbiol. 2011; 49(6):2318-20

33. Rosenblum BB, Lee LG, Spurgeon SL, Khan SH, Menchen SM, Heiner CR, et al. New dye-labeled terminators for improved DNA sequencing patterns. Nucleic Acids Res. 1997;25(22):4500-4.

34. Katoh K, Standley DM. MAFFT multiple sequence alignment software version 7: improvements in performance and usability. Mol Biol Evol. 2013, 30(4):772-80.

35. Tamura K, Peterson D, Peterson N, Stecher G, Nei M, Kumar S. MEGA5: molecular evolutionary genetics analysis using maximum likelihood, evolutionary distance, and maximum parsimony methods. Mol Biol Evol. 2011;28(10):2731-9.

36. De Graaf M, Osterhaus ADME, Fouchier RAM, Holmes EC. Evolutionary dynamics of human and avian metapneumoviruses. J Gen Virol. 2008;2018: 2933-42.

37. Yang Z, Swanson WJ. Codon-substitution models to detect adaptive evolution that account for heterogeneous selective pressures among site classes. Mol Biol Evol. 2002;19(1):49-57.

38. Yang Z. PAML 4: phylogenetic analysis by maximum likelihood. Mol Biol Evol. 2007;24(8):1586-91

39. World Health Organization (WHO). Acute respiratory infections in children: case management in small hospitals in developing countries, a manual for doctors and other senior health workers. Geneva: World Health Organisation; 1990. p. 76.

40. Vicente D, Montes M, Cilla G, Perez-yarza EG, Perez-trallero E. Differences in clinical severity between genotype a and genotype $B$ human Metapneumovirus infection in children. Clin Infect Dis. 2006;42(March):111-3.

41. Nidaira M, Taira K, Hamabata H, Kawaki T, Gushi K, Mahoe Y, et al. Molecular epidemiology of human metapneumovirus from 2009 to 2011 in Okinawa. Japan Japanese Journal of Infectious Diseases. 2012;65(4):337-40.

42. Pitoiset C, Darniot M, Huet F, Aho S, Manoha C. Human metapneumovirus genotypes and severity of disease in young children $(n=100)$ during a 7 year study in Dijon. J Med Virol. 2010;82(10):1782-9.
43. Yang C-F, Wang CK, Tollefson SJ, Piyaratna R, Lintao LD, Chu M, et al. Genetic diversity and evolution of human metapneumovirus fusion protein over twenty years. Virol J. 2009;6(1):138.

44. Li J, Ren L, Guo L, Xiang Z, Paranhos-Baccalà G, Vernet G, et al. Evolutionary dynamics analysis of human metapneumovirus subtype A2: genetic evidence for its dominant epidemic. PLoS One. 2012;7(3):1-9.

45. Padhi A, Verghese B. Positive natural selection in the evolution of human metapneumovirus attachment glycoprotein. Virus Res. 2008;131(2):121-31.

46. Peret TC, Abed Y, Anderson LJ, Erdman DD, Boivin G. Sequence polymorphism of the predicted human metapneumovirus $G$ glycoprotein. J Gen Virol. 2004 Mar 1;85(3):679-86.

47. Ngama M, Bett A, Cane PA, Medley GF, Nokes DJ, Gitahi CW, et al. Molecular evolutionary dynamics of respiratory syncytial virus group a in recurrent epidemics in coastal Kenya. J Virol. 2016;90(10):4990-5002.

48. Zlateva KT, Lemey P, Vandamme AM, Van Ranst M. Molecular evolution and circulation patterns of human respiratory syncytial virus subgroup a: positively selected sites in the attachment G glycoprotein. J Virol. 2004;78(9):4675-83.

49. Skiadopoulos MH, Biacchesi S, Buchholz UJ, Riggs JM, Surman SR, AmaroCarambot E, McAuliffe JM, Elkins WR, Claire MS, Collins PL, Murphy BR. The two major human metapneumovirus genetic lineages are highly related antigenically, and the fusion (F) protein is a major contributor to this antigenic relatedness. J Virol. 2004 Jul 1;78(13):6927-37.

50. Gunson RN, Collins TC, Carman WF. Practical experience of high throughput real time PCR in the routine diagnostic virology setting. J Clin Virol. 2006; (Apr) 1;35(4):355-67.

51. Kamau E, Agoti CN, Lewa CS, Oketch J, Owor BE, Otieno GP, et al. Recent sequence variation in probe binding site affected detection of respiratory syncytial virus group B by real-time RT-PCR. J Clin Virol. 2017:88:21-5.

52. Silaba M, Ooko M, Bottomley C, Sande J, Benamore R, Park K, et al. Articles effect of 10-valent pneumococcal conjugate vaccine on the incidence of radiologically-confirmed pneumonia and clinically-defined pneumonia in Kenyan children : an interrupted time-series analysis. Lancet Global Health. 2019:7(3):e337-46

53. Madhi SA, Klugman KP. A role for Streptococcus pneumoniae in virusassociated pneumonia. Nat Med. 2004;10(8):811-3.

54. Fathima P, Blyth CC, Lehmann D, Lim FJ, Abdalla T, De Klerk N, et al. The impact of pneumococcal vaccination on bacterial and viral pneumonia in Western Australian children: record linkage cohort study of 469589 births, 1996-2012. Clinical Infectious Disease. 2018;66(7):1075-85.

55. Hughes AL, Hughes MA. More effective purifying selection on RNA viruses than in DNA viruses. Gene. 2007 Dec 1;404(1-2):117-25.

56. Nyiro JU, Munywoki P, Kamau E, Agoti C, Gichuki A, Etyang T, et al. Surveillance of respiratory viruses in the outpatient setting in rural coastal Kenya: baseline epidemiological observations. Wellcome Open Research. 2018;3.

\section{Publisher's Note}

Springer Nature remains neutral with regard to jurisdictional claims in published maps and institutional affiliations.

\section{Ready to submit your research? Choose BMC and benefit from:}

- fast, convenient online submission

- thorough peer review by experienced researchers in your field

- rapid publication on acceptance

- support for research data, including large and complex data types

- gold Open Access which fosters wider collaboration and increased citations

- maximum visibility for your research: over $100 \mathrm{M}$ website views per year

At $\mathrm{BMC}$, research is always in progress.

Learn more biomedcentral.com/submissions 